\title{
Editorial
}

\section{Welcome to a New Journal-Journal of Genome Editing and Regulation}

\author{
Jae Yong Han ${ }^{1}$ and Jiuzhou Song ${ }^{2}$ \\ ${ }^{1}$ Department of Agricultural Biotechnology, and Research Institute of Agriculture and Life Sciences, \\ Seoul National University, Seoul 08826, Korea \\ ${ }^{2}$ Department of Animal and Avian Sciences, University of Maryland, College Park, MD 20742, USA \\ Address correspondence to Jae Yong Han, jaehan@snu.ac.kr; Jiuzhou Song, songj88@umd.edu
}

Received 25 June 2021; Accepted 25 June 2021

Copyright ( 2021 Jae Yong Han and Jiuzhou Song. This is an open access article distributed under the terms of the Creative Commons Attribution License, which permits unrestricted use, distribution, and reproduction in any medium, provided the original work is properly cited.

In the postgenomic era, decoding the mystery of the genome and understanding the roles of genes, regulatory elements, and networks are big challenges. Characterization of regulatory mechanisms will provide unique insights into genome biology, growth development, reproduction, and the evolution. The genetics of Mendelian and complex traits, and the dissection of the genome to phenome are of significant importance to basic science, human health, and agriculture; interdisciplinary research encompassing genetics, genomics, epigenetics, and systems biology is required, not only to decipher the genome, but to engineer it to functionally explore the roles of genomes. To this end, based on engineered or bacterial nucleases, genome editing technologies is a rapidly developing biotechnology area in which the nucleotide sequence of the genome of living cells is precisely changed, opening up the possibility of directly targeting and modifying genomic sequences in almost all cells and organisms.

Genome editing technologies have been developed since the late 20th century (1970s) and have globally enabled researchers to delete, insert or modify the genome of almost all living cells in vitro as well as in vivo. In recent times, ground-breaking genome editing tools such as meganucleases, zinc finger nucleases (ZFNs), transcription activator-like effector nucleases (TALENs), clustered regularly interspaced short palindromic repeats (CRISPR)/CRISPR associated nucleases (CRISPR/Cas), and base-editors were rapidly developed; enabling precise genome editing of living cells. Among these genome editing tools, CRISPR-based tools are the most widely used in recent years, because the CRISPR-mediated genome editing is simpler and faster than the other tools, and the target site is relatively less restricted. These genome editing nucleases induce double-stranded breaks (DSBs) at specific sites in the genome of the transfected cells by targeted recognition and cleavage. When the nucleases-induced
DSBs occur in cells, it can be repaired by one of the highly conserved DSB repair pathways: a nonhomologous end joining (NHEJ) repairs the break by directly rejoining the two DSB ends, and thus frequently causes insertions and deletions (indels); while a homology-directed repair (HDR) repairs the break more accurately by using the donor template. In addition, by utilizing dead cas9, which lacks nuclease function, together with specific regulators, it is possible to activate or repress the expression of specific genes, and further enable epigenetic regulation. These genome editing technologies are allowing mankind to experience a new era for the development of new biological traits, sustainable biological resources, and the control of specific diseases through the precise editing of genes in living organisms. Most importantly, the development of genome editing techniques and methods is extending our ability to elucidate the contribution of genetics to disease, biological traits, and agricultural merits by promoting the creation of more accurate cellular and animal models of pathological processes and phenotypes, which have demonstrated extraordinary potential in a variety of fields, ranging from basic research to applied biotechnology and biomedical research.

Meanwhile, since genome editing techniques require a very high level of precision, these techniques must be applied wisely and selectively. First of all, the introduction of genome editing technology must overcome the low editing efficiency that frequently shows up for cells such as stem cells. To apply the ideal genome editing technology, efficient delivery systems of various genome editing tools or direct delivery attempts at mRNA or protein levels are required. In particular, the fact that HDR repair, which is essential for precise genome editing, occurs with lower efficiency than NHEJ repair is a limitation that needs to be solved. In addition, systems to improve the editing efficiency of the target site and to eliminate off-target 
must be premised, especially in biomedical usages. Many advanced systems have been developed for this purpose; but to date, there are still many challenges and regulations are needed.

As numerous journals in the broad categories of "life sciences" have been publishing articles covering the above or more different applications, this field has been dramatically growing, including the introduction of genome editing technologies, the development of new strategies, the impact of the strategy on animals, methods to avoid offtarget effects, important discussions on animal ethics, and so on. According to the National Center for Biotechnology Information (NCBI) PubMed index, approximately 22,190 articles of various types (research articles, review articles, letters, technical notes, etc.) were published with the keyword "Genome editing" in different journals since 1977, among which approximately 4,300 articles were published since the last one year. We should believe that this number of published articles might be several folds higher when considering the articles not indexed in the PubMed. However, journals specific to "genome editing category" are only few, and this category of journals should be increased to facilitate the literature search by the scientific community and professionals working in this field.

Therefore, the Journal of Genome Editing and Regulation (JGER) aims to provide a platform for presenting and discussing all cutting-edge gene regulatory research and genome-editing. This will improve our understanding of genome to phenome, and guide the development of new strategies for decoding and annotating genomes. The journal welcomes multidisciplinary genome research submissions, including molecular biology, genetics, epigenetics, computational biology, and comparative genomics studies. Research that can develop gene regulations' impacts, editing on viruses and bacteria's genomes to improve animal and human diseases' therapeutics is highly encouraged.

The scope of our research includes, but is not limited to:

- Gene regulation at the transcriptional and posttranscriptional levels

- Genome editing technologies

- Epigenetics and sequence variations

- RNA structure and function

- Single cell technology and methods

- Computational biology

- Comparative genomics

- Chromatin structure and function

- Genomics and disease.

The journal welcomes high-quality original papers, reviews, opinions, debates, and invited editorials. As Editors-in-Chief of the Journal of Genome Editing and Regulation, we will be dedicated to disseminate peerreviewed basic and experimental research related to the above-mentioned scope of the journal.
All articles published in the Journal of Genome Editing and Regulation are open access and distributed under the Creative Commons Attribution License, which permits unrestricted use, distribution, and reproduction in any medium, provided the original work is properly cited. For more information regarding the Journal of Genome Editing and Regulation, including our distinguished Board of Associate Editors, we invite you to visit our website (http://www.bendola.com/journals/jger). We also invite you to submit manuscripts to be considered for potential publication in this new peer-reviewed Journal of Genome Editing and Regulation (JGER) and stimulate collaborating colleagues to do so.

Jae Yong Han and Jiuzhou Song Editors-in-Chief 